\title{
Wheat bran affects the site of fermentation of resistant starch and luminal indexes related to colon cancer risk: a study in pigs
}

\author{
M J A P Govers, N J Gannon, F R Dunshea, P R Gibson, J G Muir
}

\begin{abstract}
Background-Recent studies suggest that resistant starch (effective in producing butyrate and lowering possibly toxic ammonia) is rapidly fermented in the proximal colon; the distal colon especially would, however, benefit from these properties of resistant starch.

Aims-To determine whether wheat bran (a rich source of insoluble non-starch polysaccharides), known to hasten gastrointestinal transit, could carry resistant starch through to the distal colon and thus shift its site of fermentation.

Methods-Twenty four pigs were fed four human type diets: a control diet, or control diet supplemented with resistant starch, wheat bran, or both. Intestinal contents and faeces were collected after two weeks.

Results-Without wheat bran, resistant starch was rapidly fermented in the caecum and proximal colon. Supplementation with wheat bran inhibited the caecal fermentation of resistant starch, resulting in an almost twofold increase (from 12.9 (2.5) to $20.5(2.1) \mathrm{g} / \mathrm{day}, \mathrm{p}<0.05$ ) in resistant starch being fermented between the proximal colon and faeces. This resulted in higher butyrate $(133 \%, p<0.05)$ and lower ammonia $(81 \%, p<0.05)$ concentrations in the distal colonic regions.

Conclusions-Wheat bran can shift the fermentation of resistant starch further distally, thereby improving the luminal conditions in the distal colonic regions where tumours most commonly occur. Therefore, the combined consumption of resistant starch and insoluble non-starch polysaccharides may contribute to the dietary modulation of colon cancer risk. (Gut 1999;45:840-847)
\end{abstract}

Keywords: resistant starch; non-starch polysaccharides; colonic fermentation; butyrate; ammonia; colon cancer risk

Epidemiological studies suggest that dietary fibre is protective against colorectal cancer. ${ }^{1}$ The physiological effects of non-starch polysaccharides (NSP), the major component of dietary fibre, in the colon are dependent on their physicochemical properties. Water soluble NSP, including guar gum and pectin, are viscous and rapidly fermentable in the proximal colon. Water insoluble NSP, such as wheat bran, are non-viscous and are only slowly fermented along the colon. McIntyre et al have shown that different fibres have different regional effects on fermentation related indexes $^{2}$ and on tumour formation ${ }^{3}$ in a rat model, with insoluble NSP showing an inhibiting effect on tumorigenesis.

Several mechanisms by which insoluble NSP are thought to exert their protective effect on colorectal carcinogenesis have been proposed. ${ }^{1}$ Fermentation of NSP by the colonic microflora produces short chain fatty acids (SCFA), including butyrate, which plays an important role in maintaining the health and integrity of the colonic epithelium. ${ }^{4}$ Colonic fermentation lowers luminal $\mathrm{pH}$, which in turn has a number of favourable effects. ${ }^{5}$ Increased carbohydrate fermentation can divert luminal ammonia, shown to be toxic to the colonic epithelium, ${ }^{6}$ into de novo synthesis of bacterial protein. ${ }^{7}$ Furthermore, insoluble NSP increase the bulk of luminal contents, which will dilute possible toxins and carcinogens, increase the transit of digesta through the colon, ${ }^{8}$ and, in turn, reduce the contact time of harmful compounds with the colonic mucosa.

In recent years, a considerable number of studies have focused on the importance of resistant starch (RS) as a substrate for colonic fermentation. RS can be subdivided in three major types: physically trapped starch (RS1, for example, in coarse grains), resistant starch granules (RS2, for example, high amylose corn, raw potato flour), and retrograded starch (RS3, for example, cooked and cooled potato). ${ }^{9}$ In reaching the colon undigested, RS can be fermented by the colonic flora. The physiological effects of RS in the colon may, therefore, be similar to those of NSP. In vitro, ${ }^{10}$ animal, ${ }^{11} 12$ and human ${ }^{13}$ studies have shown that fermentation of RS generates relatively more butyrate than NSP. A study in our laboratory indicated that RS behaves more like soluble NSP than insoluble NSP, in that it will be rapidly fermented in the proximal colon. ${ }^{14}$ In line with these observations, Young et al have shown that RS, like soluble NSP, enhances formation of aberrant crypts in the rat. ${ }^{15}$ In humans, RS has been shown to increase faecal bulk and SCFA concentrations, and to lower faecal $\mathrm{pH}$ and concentrations of ammonia and phenols. ${ }^{13}$ 16-20 The effects of RS on faecal indexes are not consistent, however, and may be dependent on the site of RS fermentation in the large bowel.

Abbreviations used in this paper: NSP, non-starch polysaccharide; RS, resistant starch; SCFA, short chain fatty acids. 
Table 1 Composition of the experimental diets ( $g / 40 \mathrm{MF}$ )

\begin{tabular}{lllll}
\hline Ingredient & Control diet & RS diet & NSP diet & RS+NSP diet \\
\hline Butter $^{\mathrm{a}}$ & 160 & 160 & 160 & 160 \\
Beef tallow $^{\mathrm{b}}$ & 120 & 120 & 120 & 120 \\
Vegetable oil $^{\mathrm{c}}$ & 110 & 110 & 110 & 110 \\
Whey protein concentrate $^{\mathrm{d}}$ & 320 & 310 & 300 & 290 \\
Blood meal $^{\mathrm{b}}$ & 170 & 170 & 170 & 170 \\
Sugar $^{\mathrm{e}}$ & 470 & 470 & 470 & 470 \\
Corn flour $^{\mathrm{f}, \mathrm{g}}$ & 450 & 570 & 450 & 570 \\
Low amylose corn kernels $^{\mathrm{f}, \mathrm{h}}$ & 220 & 0 & 220 & 0 \\
High amylose corn kernels $^{\mathrm{f}, \mathrm{i}}$ & 60 & 280 & 60 & 280 \\
Wheat brann $^{\mathrm{f}, \mathrm{h}}$ & 0 & 0 & 140 & 140 \\
Corn bran $^{\mathrm{f}, \mathrm{h}}$ & 60 & 40 & 60 & 40 \\
Salt & 5 & 5 & 5 & 5 \\
Vitamin and mineral mixture $^{\mathrm{j}}$ & 5 & 5 & 5 & 5 \\
Celite & 50 & 50 & 50 & 50 \\
As analysed & & & & \\
RS & 41 & 122 & 39 & 119 \\
NSP & 83 & 84 & 137 & 138 \\
Digestible starch & 501 & 496 & 507 & 502 \\
\hline
\end{tabular}

${ }^{\mathrm{a} W e s t e r n ~ S t a r}$ (Melbourne, Victoria, Australia); ${ }^{\mathrm{b} P e e r l e s s ~(B r a y b r o o k, ~ V i c t o r i a, ~ A u s t r a l i a) ; ~}{ }^{\mathrm{C} C r i s c o}$ (Mascot, New South Wales, Australia); ${ }^{\mathrm{d} U n i t e d ~ M i l k ~ T a s m a n i a ~(D e v o n p o r t, ~ T a s m a n i a, ~ A u s t r a l i a) ; ~}$ ${ }^{\mathrm{e}} \mathrm{CSR}$ (Yarraville, Victoria, Australia); ${ }^{\mathrm{f}}$ kindly provided by Starch Australasia Ltd (Lane Cove, New South Wales, Australia); ${ }^{\mathrm{g}}$ wheaten corn flour, gelatinised in 5 litres boiling water; ${ }^{\text {h}}$ particle size $\leqslant 1$ $\mathrm{mm}$; 'particle size $\leqslant 3 \mathrm{~mm}$; ${ }^{\text {provides }}(\mathrm{mg} / 40 \mathrm{MJ}$ ): retinol 16, cholecalciferol 0.21 , $\alpha$ tocopherol 55 , menadione 1.5 , riboflavin 8.3 , nicotinic acid 41.3 , pantothenic acid 13.8 , pyrodoxine 2.8 , biotin 1.4, choline 2750, cyanocobalamin 0.043, Fe 220, Zn 138, Mn 55, Cu 16.5, I 0.55, Se 0.25, Propharma (Dandenong, Victoria, Australia); ${ }^{k}$ Diatomaceous earth (Celite Corporation, Lompoc, California, USA).

RS, resistant starch; NSP, non-starch polysaccharide.

If the protective effects of undigested carbohydrates in the colon are indeed related to the site of their fermentation, ${ }^{3}$ it would be desirable to shift the fermentative process of RS to the more distal regions, as the majority of colonic tumours in humans occur in the distal colon and rectum. Insoluble NSP are known to increase the transit of digesta through the colon, ${ }^{8}$ and therefore may be able physically to push the RS further along the colon. In human diets, RS and NSP coexist, but little is known about the effects of NSP on the fermentation of RS. Key and Mathers ${ }^{21}$ and Young and colleagues $^{15}$ found that insoluble NSP increased faecal starch excretion in rats. In addition, the latter group showed that the enhanced tumorigenesis in the distal colon by RS was suppressed by adding wheat bran to the diet. These studies suggest that insoluble NSP indeed carry RS through to the distal colon. However, to date, no study has systematically and quantitatively looked at the site of fermentation of RS and NSP by actually monitoring the disappearance of these substrates along the colon. Therefore, the aim of the present study was to determine the exact site of fermentation of RS and insoluble NSP and the possible interaction between the two. The separate and combined effects of RS and NSP on fermentation related indexes along the gastrointestinal tract, including bulking, luminal $\mathrm{pH}$, SCFA and ammonia concentrations, were also studied.

Determining the site of colonic fermentation requires invasive procedures, which are for obvious reasons difficult to undertake in humans. The pig has been shown to be a good model for nutrition studies, as in terms of gastrointestinal anatomy, physiology, and nutrient requirements it is more similar to the human than all other domesticated animal species. ${ }^{22}{ }^{23}$ While rats are primarily caecal fermenters, pigs and humans are essentially colon fermenters. Furthermore, the composition of the colonic flora is comparable in pigs and humans. $^{23}$

\section{Methods}

ANIMALS

Twenty four male pigs (Large White $\times$ Landrace, Pig Research and Training Centre, Werribee, Victoria, Australia) were used; they were aged four months and weighed a mean of $61 \mathrm{~kg}$ (SEM $1 \mathrm{~kg}$, range 56-65) at the start of the study. The pigs were divided into four groups of six animals each, matched for body weight, and housed in individual pens for the entire duration of the study (12 hour light/dark cycle, temperature $\left.18-24^{\circ} \mathrm{C}\right)$. The animals had free access to tap water via nipple drinkers. The study protocol was approved by the Animal Experimentation Ethics Committees of both the Victorian Institute of Animal Science and Deakin University.

EXPERIMENTAL DIETS

Four semipurified experimental diets were designed, differing only in the amount of RS and NSP. The amount of digestible starch was kept constant. The macronutrient composition of the diet was based on a Western style human diet, and comprised $18 \%$ of energy from protein, $35 \%$ from fat, and $47 \%$ from digestible carbohydrate (of which $20 \%$ was from monosaccharides). The contribution of fermentation to the energy content of the diets was not taken into account. The dietary fatty acid composition was also similar to that of the human diet. Animals were subjected to a slightly restricted energy intake of $40 \mathrm{MJ} /$ day (about $80 \%$ of usual energy intake) to ensure the complete consumption of their daily ration.

Table 1 gives the composition of the diets. The concentrations of RS and NSP in the control diet were chosen to be $40 \mathrm{~g}$ RS and $80 \mathrm{~g}$ $\mathrm{NSP} / 40 \mathrm{MJ}$ to prevent constipation. To increase the amount of RS in the diet, coarse high amylose corn kernels (40 g RS/100 g, particle size $\leqslant 3 \mathrm{~mm}$ ), were substituted for low amylose corn kernels (4 g RS/100 g), providing a combination of RS1 and RS2. This increased the dietary RS concentration to $120 \mathrm{~g} / 40 \mathrm{MJ}$. Wheat bran was added to increase the amount of insoluble NSP in the diets to $140 \mathrm{~g} / 40 \mathrm{MJ}$. The rationale for choosing these concentrations was that similar densities are achievable in the human diet (30 $\mathrm{g}$ RS and $35 \mathrm{~g} \mathrm{NSP} / 10 \mathrm{MJ}$ ) and hence they are physiologically relevant to the human situation. Concomitant (but unwanted) changes in the amounts of digestible starch, NSP, and protein caused by the RS and NSP supplements were compensated for by adjusting the amounts of corn flour, corn bran, and whey protein. Celite was added to the diets to enable calculation of daily flow rates (see later).

All ingredients, except the corn flour, were mixed until homogeneous to form a premix and stored at $4^{\circ} \mathrm{C}$. On a daily basis, the corn flour for each pig was gelatinised in 5 litres of boiling tap water and cooled overnight. This procedure reduced the amount of RS in the corn flour to less than $2 \%$. The corn flour was 
mixed thoroughly with the precise amount of diet premix and fed to the animals as a single meal in the morning.

\section{COLLECTION OF FAECES AND INTESTINAL}

CONTENTS

A two week run in period was chosen to give the colonic flora sufficient time for adaptation to the diets. ${ }^{24}$ In the mornings on day 15,16 , and 17 , fresh faecal samples were collected onto dry ice immediately after defecation and stored at $-40^{\circ} \mathrm{C}$ until processing. On days 18 and 19, three pigs from each group were slaughtered on each day. Pigs were weighed and subsequently fed their daily ration in two portions, approximately 3.5 and two hours before slaughter, to ensure the presence of digesta in the small intestine and to maintain normal postprandial gastrointestinal motility and transit. Slaughter took place at an experimental abattoir (Meat Research and Training Centre, Werribee, Victoria, Australia). Pigs were killed at 20 minute intervals in such order that the effect of time was minimised across groups. After stunning with $\mathrm{CO}_{2}$, the animals were bled, dehaired, and subsequently the gastrointestinal tract was excised. Immediately after excision, the caecum was tied off from the terminal ileum and proximal colon, and caecal contents were collected. The contents of the most distal $4 \mathrm{~m}$ of the small intestine, representing the terminal ileum, were then collected. Finally, the large bowel was unravelled and divided into three equal lengths, representing proximal, middle, and distal colon, and the contents of each section were collected. All intestinal contents were placed on dry ice within 15 minutes of excision of the gut, and stored at $-40^{\circ} \mathrm{C}$ until processing.

\section{PROCESSING OF INTESTINAL CONTENTS AND}

FAECES

Intestinal contents and faeces were thawed overnight at $4^{\circ} \mathrm{C}$. Faeces from each of the three days were processed and analysed separately. Samples were weighed and subsequently homogenised on ice. Aliquots for analysis of SCFA (1:3 diluted in saline) and ammonia were stored at $-40^{\circ} \mathrm{C}$. Aliquots were freeze dried to constant weight and the percentage of dry matter was calculated. An aliquot of approximately $5 \mathrm{~g}$ was brought to $37^{\circ} \mathrm{C}$ and $\mathrm{pH}$ was measured using a protein resistant electrode. An average of three readings at different sites was taken. Because of the dry consistency of samples from the distal colon and faeces, these samples were homogenised in $5 \mathrm{ml}$ distilled water prior to measuring $\mathrm{pH}$. Control experiments with samples from middle colon and relatively wet samples from distal colon showed that the $\mathrm{pH}$ was not affected by an up to threefold dilution of the digesta.

BIOCHEMICAL ANALYSES

The relevant dietary ingredients and the complete diets were analysed (after cooling) for RS using the method of Muir and O'Dea, ${ }^{25}$ with the modifications that chewing was omitted and that the RS containing residues were freeze dried and analysed for total starch using a commercially available kit (Megazyme Australia, Warriewood, New South Wales, Australia). Diets and freeze dried digesta were analysed for total starch (as above) and for total NSP according to the colorimetric method of Englyst and Cummings. ${ }^{26}$ The digestible starch content in the diets was calculated as the difference between total starch and RS. SCFA were analysed by gas chromatography as described previously. ${ }^{16}$ Total SCFA were calculated as the sum of acetate, propionate, and butyrate. Ammonia concentrations were measured according to the colorimetric method of Lin and Visek. ${ }^{27}$

\section{CALCULATION OF DAILY FLOW RATES}

The flow of digesta, substrates, and metabolites in the intestinal sections was calculated by reference to the indigestible, non-absorbable marker Celite (diatomaceous earth). ${ }^{28}$ The determination of Celite content in digesta was based on the method for acid insoluble ash of Van Keulen and Young ${ }^{29}$ with modifications. Freeze dried samples were reduced to ash over two hours at $650^{\circ} \mathrm{C}$. The ash was subsequently dissolved in $2 \mathrm{M} \mathrm{HCl}$ and incubated in a boiling water bath for 30 minutes. After centrifugation (five minutes, $2000 \mathrm{~g}$ ), the supernatant (containing the acid soluble ash) was discarded and the pellet (containing the acid insoluble ash) washed in $2 \mathrm{M} \mathrm{HCl}$ and freeze dried to constant weight. Control experiments showed that the amount of acid insoluble ash in digesta without Celite was negligible, hence pellet weight was considered to be Celite weight. Recovery of added Celite was greater than $96 \%$. Assuming that the Celite was consumed completely and homogeneously, the flow rates ( $\mathrm{g} /$ day) of digesta in a given section were calculated as the daily intake of Celite divided by the ratio Celite:digesta in that section, as described by Goodlad and Mathers. ${ }^{30}$ The flow rates of substrates and metabolites were calculated as concentration $\times$ flow rate of digesta in a given section.

\section{STATISTICS}

Data were analysed using the statistical software package SPSS for Windows 6.0 (SPSS Inc., Chicago, Illinois, USA). Results are given as the mean (SEM) for six animals per group, unless indicated otherwise. Data of faeces of the three collection days were analysed separately. Only minor differences were seen across days, and therefore the average of the three days is presented. Differences across groups were analysed by one way analysis of variance (ANOVA). If $\mathrm{p}<0.05$, intergroup comparisons were made using the least significant difference test. To determine whether the combined effects of RS and NSP were additive or synergistic, a two way ANOVA, with RS and NSP as the independent factors, was used. A significant $(p<0.05)$ interaction term was interpreted as a positive interaction between RS and NSP. Relations between variables were determined by Pearson's correlation and by stepwise multiple regression analysis, with $\mathrm{p}<0.05$ regarded as significant. 

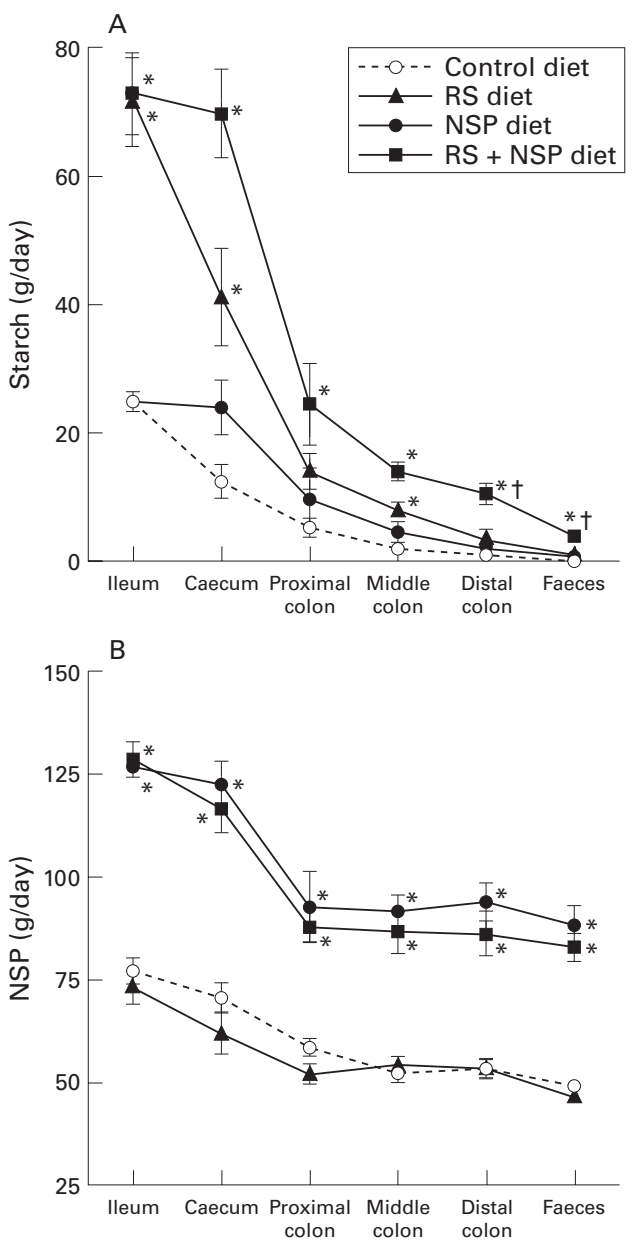

Figure 1 Effects of diets supplemented with resistant starch (RS) and/or non-starch polysaccharide (NSP) on daily amounts of $(A)$ starch and $(B)$ NSP in intestinal contents and faeces. ${ }^{*}$ Significantly different from control group (one way ANOVA, $p<0.05$ ). +Significant positive interaction between RS and NSP (two way ANOVA, $p<0.05$ ).

\section{Results}

FOOD INTAKE AND BODY WEIGHTS

All pigs consumed their daily ration of food usually within an hour, except for two animals in the control group, who initially ate less. In the week prior to collections, all pigs consumed all food provided. The final body weight of the pigs was significantly affected by diet $(77.6$ (1.9), 80.9 (1.9), 81.5 (1.9), and 82.9 (1.3) kg for the control, RS, NSP, and RS+NSP groups,

Table 2 Flow rates of digesta and faeces (g/day)

\begin{tabular}{lllll}
\hline & Control diet & RS diet & NSP diet & RS+NSP diet \\
\hline Wet weight & $1908(257)^{\mathrm{a}}$ & $1919(130)^{\mathrm{a}}$ & $2500(249)^{\mathrm{b}}$ & $2642(214)^{\mathrm{b}}$ \\
Ileum & $1517(158)^{\mathrm{a}}$ & $1649(168)^{\mathrm{a}}$ & $2080(114)^{\mathrm{ab}}$ & $2473(345)^{\mathrm{b}}$ \\
Caecum & $798(71)^{\mathrm{a}}$ & $813(46)^{\mathrm{a}}$ & $1067(127)^{\mathrm{ab}}$ & $1166(141)^{\mathrm{b}}$ \\
Proximal colon & $622(19)^{\mathrm{a}}$ & $682(46)^{\mathrm{a}}$ & $918(37)^{\mathrm{b}}$ & $1031(90)^{\mathrm{b}}$ \\
Middle colon & $530(20)^{\mathrm{a}}$ & $631(54)^{\mathrm{a}}$ & $916(47)^{\mathrm{b}}$ & $1052(78)^{\mathrm{b}}$ \\
Distal colon & $383(9)^{\mathrm{a}}$ & $443(3)^{\mathrm{a}}$ & $664(41)^{\mathrm{b}}$ & $799(35)^{\mathrm{c}}$ \\
Faeces & & & & \\
Dry weight & $355(19)^{\mathrm{a}}$ & $398(24)^{\mathrm{ab}}$ & $424(17)^{\mathrm{b}}$ & $462(14)^{\mathrm{c}}$ \\
Ileum & $265(13)^{\mathrm{a}}$ & $305(19)^{\mathrm{a}}$ & $393(16)^{\mathrm{b}}$ & $453(29)^{\mathrm{c}}$ \\
Caecum & $224(13)^{\mathrm{a}}$ & $226(7)^{\mathrm{a}}$ & $292(29)^{\mathrm{b}}$ & $295(18)^{\mathrm{b}}$ \\
Proximal colon & $206(5)^{\mathrm{a}}$ & $222(14)^{\mathrm{a}}$ & $282(18)^{\mathrm{b}}$ & $293(16)^{\mathrm{b}}$ \\
Middle colon & $198(4)^{\mathrm{a}}$ & $219(12)^{\mathrm{a}}$ & $277(11)^{\mathrm{b}}$ & $296(11)^{\mathrm{b}}$ \\
Distal colon & $192(5)^{\mathrm{a}}$ & $197(8)^{\mathrm{a}}$ & $260(11)^{\mathrm{b}}$ & $281(6)^{\mathrm{b}}$ \\
Faeces & & &
\end{tabular}

Results expressed as mean (SEM), $\mathrm{n}=6$. Values in the same row not sharing the same superscripts are significantly different $(\mathrm{p}<0.05)$.

RS, resistant starch; NSP, non-starch polysaccharide.

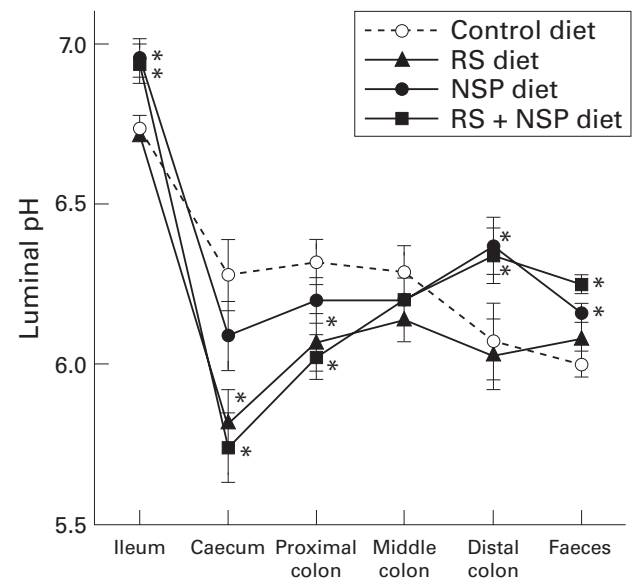

Figure 2 Effects of diets supplemented with resistant starch (RS) and/or non-starch polysaccharide (NSP) on pH of intestinal contents and faeces. * Significantly different from control group (one way ANOVA, $p<0.05$ ).

respectively), with the RS+NSP group having a significantly higher body weight than the control group. This is probably due to a slightly higher energy content of the RS+NSP diet, caused by the additional energy from fermentable substrates.

\section{BREAKDOWN OF RS AND NSP}

Figure 1 shows the levels of starch and NSP in the five intestinal sections and in faeces. The daily amount of starch in ileal digesta represented approximately $60 \%$ of the dietary intake of RS and was unaffected by the presence of insoluble NSP in the diet. The daily ileal excretion of NSP was approximately $90 \%$ of dietary intake and was also not affected by the presence of starch. In the control and RS groups, starch was fermented rapidly in the proximal regions of the colon, with negligible starch being excreted in faeces (fig 1A). Addition of NSP to the control and RS diets delayed starch fermentation in the caecum, which, in the RS+NSP group, resulted in higher amounts of starch reaching the distal regions of the large bowel and the faeces. Addition of NSP to the RS diet increased the amount of starch fermented between the proximal colon and faeces from 12.9 (2.5) to 20.5 (2.1) g/day. A positive interaction between RS and NSP with regard to starch excretion was seen in distal colon and faeces. Figure 1B shows that the NSP levels decreased only in the caecum and proximal colon. Dietary RS affected neither the site nor the level of fermentation of NSP. In all groups, approximately $35 \%$ of NSP entering the large bowel was fermented.

FLOW RATES OF DIGESTA AND FAECES

Table 2 shows the flow rates of digesta along the gastrointestinal tract. In all groups, the daily flow of wet digesta decreased rapidly in the caecum and proximal colon and then gradually reduced further in the distal regions of the large bowel. While RS had minimal effect on the wet weight of intestinal contents, NSP increased the mass in most sections. The quantitative contribution of available fermentable carbohydrate in the large bowel to 

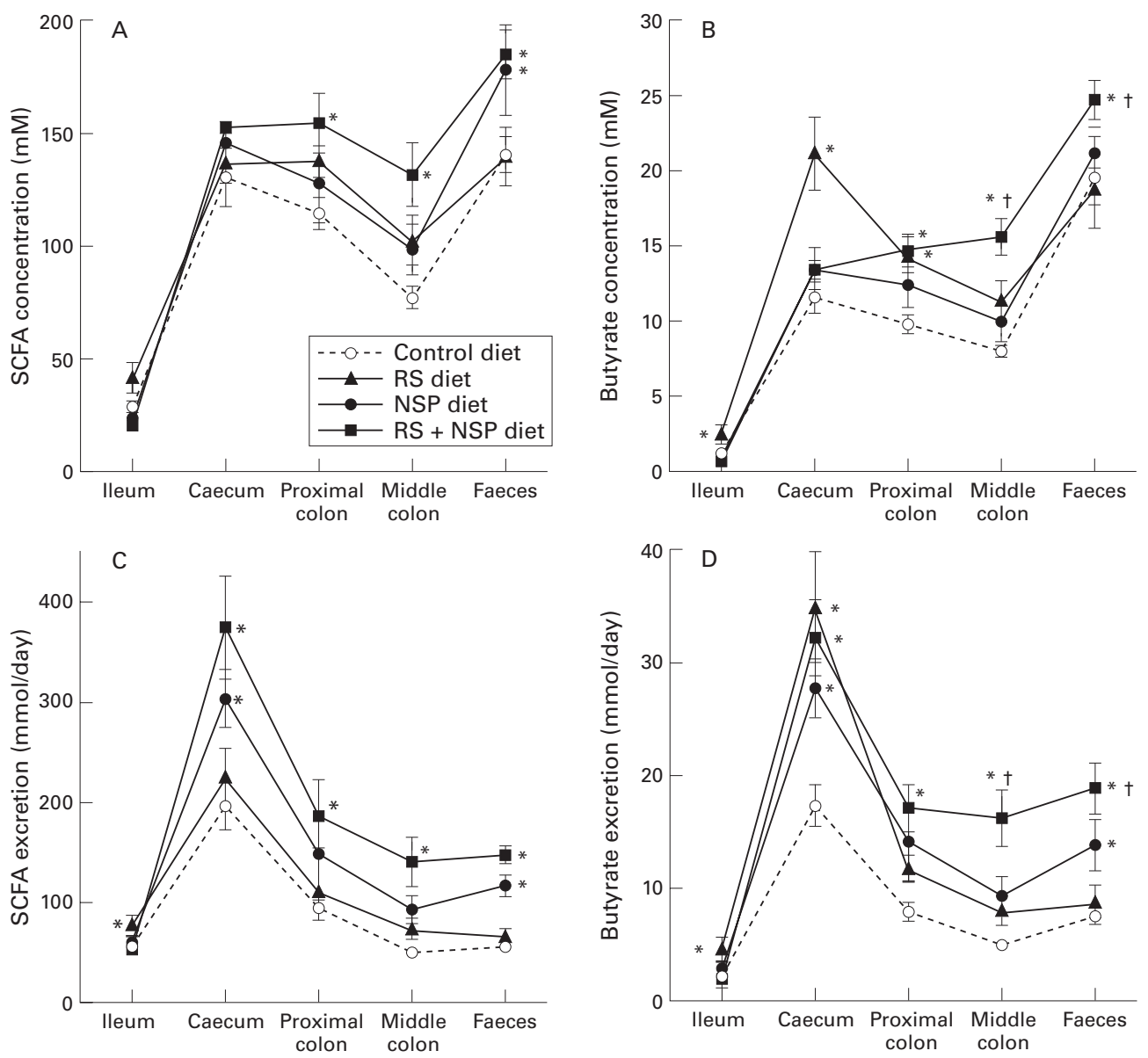

Figure 3 Effects of diets supplemented with resistant starch (RS) andlor non-starch polysaccharide (NSP) on concentrations of (A) total short chain fatty acids (SCFA) and (B) butyrate, and on daily amounts of (C) total SCFA and (D) butyrate in intestinal contents and faeces. ${ }^{\star}$ Significantly different from control group (one way ANOVA, $p<0.05$ ). tSignificant positive interaction between RS and NSP (two way ANOVA, $p<0.05$ ).

faecal bulking was determined by stepwise multiple regression analysis. NSP and RS together explained $74 \%\left(r^{2}\right)$ of the variance in faecal wet weight, of which $68 \%$ was contributed by NSP alone.

The mean increase in ileal dry matter in the supplemented groups corresponded to the higher ileal excretion of starch and/or NSP in the respective groups. Similar to wet digesta, a rapid decrease in dry matter was observed proximally, followed by only a slight reduction distally. Again, dry matter was not affected by RS, but strongly increased by NSP. The moisture content of the digesta showed no significant differences across groups up to the proximal colon (data not shown). From the middle colon onwards, NSP increased the moisture content of the digesta, while RS had no signifi-

Table 3 Concentrations and flow rates of total short chain fatty acids (SCFA) and butyrate in distal colon

\begin{tabular}{lllll}
\hline & $\begin{array}{l}\text { Control diet } \\
(n=5)\end{array}$ & $\begin{array}{l}\text { RS diet } \\
(n=4)\end{array}$ & $\begin{array}{l}\text { NSP diet } \\
(n=4)\end{array}$ & $\begin{array}{l}\text { RS+NSP diet } \\
(n=1)^{*}\end{array}$ \\
\hline $\begin{array}{l}\text { Concentration (mM) } \\
\quad \begin{array}{l}\text { Total SCFA } \\
\text { Butyrate }\end{array}\end{array}$ & $95(9)$ & $111(6)$ & $116(31)$ & 146 \\
$\begin{array}{l}\text { Fow rate (mmol/day) } \\
\quad \text { Total SCFA }\end{array}$ & $50(6)$ & $14.4(1.0)$ & $12.4(2.8)$ & 18.5 \\
$\quad$ Butyrate & $6.8(0.8)$ & $65(5)$ & $102(19)$ & 140 \\
\hline
\end{tabular}

Results expressed as mean (SEM). No significant differences across groups were observed. ${ }^{\star}$ Average of triplicate analysis of one sample only (other samples lost due to technical error). RS, resistant starch; NSP, non-starch polysaccharide. cant effect. The moisture content in faeces was $50(1) \%, 54(2) \%, 61(1) \%$, and 64 (2) \% for control, RS, NSP, and RS+NSP groups, respectively. No positive interactions between RS and NSP were observed with regard to wet weight, dry weight, or moisture content.

INTESTINAL AND FAECAL $\mathrm{pH}, \mathrm{SCFA}$, AND

AMMONIA

Figure 2 shows the $\mathrm{pH}$ of intestinal contents and faeces. From the ileum to the caecum, a substantial fall in $\mathrm{pH}$ was observed in all groups. RS, but not NSP, significantly lowered $\mathrm{pH}$ in samples from the caecum and proximal colon. In the middle colon, no differences across groups were observed, while in the distal colon and faeces NSP, but not RS, significantly increased $\mathrm{pH}$.

Figure 3 shows the concentrations and daily flow of total SCFA and butyrate in the intestinal contents and faeces. Because of an incomplete dataset for distal colon, these values were omitted from the graphs and are presented in table 3. Total SCFA concentrations (fig 3A) were low in ileal digesta, and increased more than threefold in the caecum. Significant differences across groups were not seen. In the middle colon, the SCFA concentrations decreased in all groups, but the RS+NSP group maintained higher levels of SCFA. RS substantially increased the caecal 


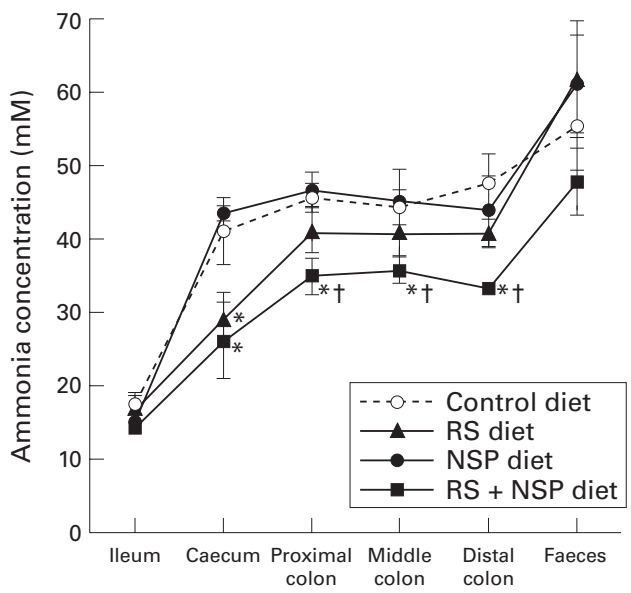

Figure 4 Effects of diets supplemented with resistant starch (RS) and/or non-starch polysaccharide (NSP) on concentrations of ammonia in intestinal contents and faeces. *Significantly different from control group (one way ANOVA, $p<0.05)$. + Significant positive interaction between RS and NSP (two way ANOVA, $p<0.05$ ).

concentration of butyrate (fig 3B). While this concentration in the RS group fell rapidly after the caecum and was not different from the control group from the middle colon onwards, the RS+NSP group showed increasing levels. A positive interaction between RS and NSP was observed in the middle colon and faeces.

Dietary NSP increased the daily flow of total SCFA in the caecum and faeces (fig 3C), while the RS+NSP diet had higher amounts in all sections compared with the control group. All supplemented diets increased the daily flow of butyrate in the caecum (fig 3D) and the RS+NSP diet maintained higher levels of butyrate in the distal regions of the colon. A positive interaction between RS and NSP was seen in middle colon and faeces on butyrate excretion. In the caecum, the relative contribution of butyrate to total SCFA was increased by dietary RS (16 (2)\%) compared with the other groups (all 9 (1)\%), at the expense of acetate. No differences across groups were seen with respect to the relative contribution of individual SCFA in all other sections (data not shown).

Figure 4 shows the ammonia concentration in intestinal and faecal samples. Ammonia concentrations increased rapidly in the proximal regions of the large bowel and levelled off in the distal colon, with a final rise in faeces. RS decreased ammonia levels in the caecum, while NSP showed no significant effect on ammonia concentrations. The RS+NSP group had significantly lower ammonia levels from the caecum to the distal colon. In the proximal, middle, and distal colon, a positive interaction between RS and NSP was observed.

Figure 5 depicts the relation between SCFA concentration and $\mathrm{pH}$ in intestinal and faecal samples. A strong inverse correlation $(r=-0.84$, $\mathrm{n}=96, \mathrm{p}<0.001$ ) was found between SCFA and $\mathrm{pH}$ up to the middle colon, whereas samples from distal colon and faeces showed no correlation. A weaker inverse correlation $(r=-0.44$, $\mathrm{n}=96, \mathrm{p}<0.001)$ was found between ammonia and $\mathrm{pH}$ up to the middle colon (graph not shown), with again no relation in distal colon

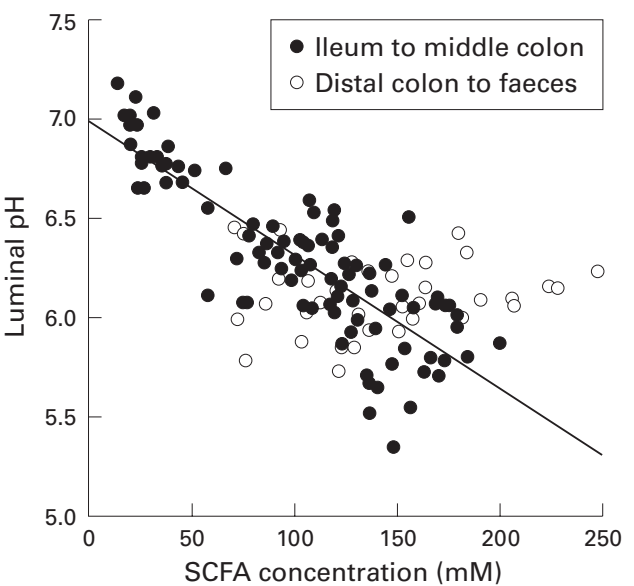

Figure 5 Relation between total short chain fatty acid (SCFA) concentration and $p H$ in intestinal and faecal samples. Ileum to middle colon: $r=-0.84, n=96, p<0.001$; distal colon and faeces: $r=+0.10, n=38, N S$. Individual sections: ileum: $r=-0.52, p<0.001$; caecum: $r=-0.60$, $p<0.001$; proximal colon: $r=-0.72, p<0.001$; middle colon: $r=-0.40, p<0.001$; distal colon: $r=-0.05, N S$; faeces: $r=+0.28$, NS (Pearson's correlation).

and faeces. When SCFA and ammonia were entered in a stepwise multiple regression analysis, SCFA concentration was the only significant predictor of luminal $\mathrm{pH}$ up to the middle colon $\left(r^{2} 71 \%\right)$. No predictors were identified in distal colon and faeces.

\section{Discussion}

Colonic fermentation of RS is known to produce more butyrate than NSP fermentation, ${ }^{10-13}$ and also to be effective in reducing luminal ammonia concentrations. ${ }^{17} 31$ Both these properties of RS are considered beneficial to the colonic epithelium, ${ }^{4}{ }^{6}$ and are thought to be possible mechanisms by which dietary starch may be protective against colon cancer. $^{32}$ The present study confirms and extends previous studies ${ }^{143}$ showing that RS from high amylose corn is rapidly fermented in the proximal colon, resulting in higher butyrate and lower ammonia concentrations in the proximal regions of the colon. More importantly, our study clearly shows, to our knowledge for the first time, that insoluble NSP can shift the fermentation of RS further distally, thereby maintaining higher butyrate and lower ammonia concentrations in the distal regions of the colon, where most tumours occur. As NSP alone had little effect on these indexes, such favourable changes in distal luminal contents can be attributed to the shifted site of RS fermentation. These findings are almost certainly physiologically relevant to humans, as the mixture of RS and insoluble NSP is common in human diets, the densities used are readily achieved in the human diet, and the physiology of the pig large bowel is very similar to that of humans.

SITE SPECIFIC FERMENTATION OF RS AND NSP While the ileal excretion of NSP almost equalled dietary intake, ileal starch represented only $60 \%$ of RS intake in each group (fig 1). This indicates that the in vitro assay for measurement of RS, as developed for 
humans, ${ }^{25}$ overestimates the amount of starch escaping digestion in the pig small intestine. This is supported by a recent observation by Heijnen and Beynen, ${ }^{34}$ who also showed that the analysed amounts of RS2 in ileal digesta of pigs deviated more from the in vitro measurement by Englyst and colleagues ${ }^{9}$ than RS3 levels. In the present study, coarse high amylose corn (particle size $\leqslant 3 \mathrm{~mm}$ ) was used, in which at least $30 \%$ of total RS is RS1 (physically trapped starch). Our preliminary experiments indicated that the analysed amounts of RS2 (in high amylose corn; $\leqslant 1 \mathrm{~mm}$ ) deviated more from the in vitro measurement than the mixture of RS1 and RS2 that was used in the present study. Combination of these findings suggests that the used in vitro RS assays ${ }^{95}$ may not be appropriate for an accurate prediction of RS levels in pig studies.

Supplemental NSP inhibited caecal starch breakdown almost completely (fig 1). By carrying RS further along the colon, addition of wheat bran to the RS diet almost doubled the amount of starch fermented distally and also resulted in substantial excretion of starch in faeces. This confirms and explains previous observations that additional NSP increased the faecal starch excretion. ${ }^{15}{ }^{21}$ A likely mechanism for this effect is NSP induced acceleration of the transit of digesta through the caecum. ${ }^{21} \mathrm{~A}$ recent study in humans showed that, while NSP from wheat bran was very effective in increasing transit, this was delayed by RS2 from banana. ${ }^{18}$ An alternative mechanism for the delayed starch fermentation may be increased substrate availability. This seems unlikely however, as the fermentation of NSP was not delayed by the increased substrate levels. Goodlad and Mathers ${ }^{35}$ have already shown that supplemental RS in pigs does not alter the amount of fermentation of wheat NSP. A new finding in the present study is that the site of NSP fermentation is also not affected by RS. These results differ from studies in humans that suggest that RS may have a sparing effect on NSP breakdown. ${ }^{18}$ This difference may be explained by the greater large bowel capacity of pigs relative to humans. ${ }^{22}$ Remarkably, NSP from wheat bran fermented only proximally and the extent of fermentation appeared independent of dietary intake. This suggests that the physical structure of the wheat fibre allows only limited breakdown by colonic bacteria, as suggested previously. ${ }^{36}$

EFFECTS OF FERMENTATION ON BULK AND OTHER FERMENTATION RELATED INDEXES

NSP is a potent bulking agent (table 2), most likely due to the large fraction of unfermented NSP and to its water holding capacity. ${ }^{36}$ Our data suggest that the latter property of NSP becomes critically important in the distal regions of the colon, as the moisture content of digesta was significantly increased by NSP from the middle colon onwards. Several human studies, ${ }^{16}{ }^{18-20}$ but not all, ${ }^{37}$ have shown that RS increases faecal output. In the present study, there was no effect of RS on faecal bulking. This may be dose related since, due to the unforseen breakdown of RS in the small intes- tine, the amount of supplemental RS reaching the colon was relatively low (50 g/40 MJ) when compared with that used in the positive human studies, ${ }^{16}{ }^{18-20}$ but similar to that applied in the study reporting no effect on faecal bulk. ${ }^{37}$

The colonic $\mathrm{pH}$ of the $\mathrm{RS}+\mathrm{NSP}$ group followed the RS group proximally and the NSP group distally (fig 2). It is not clear why the RS+NSP group showed such a substantial drop in caecal $\mathrm{pH}$ when the disappearance of RS and NSP was only minimal (fig 1). A possible explanation is that small intestinal breakdown products, such as maltose and oligosaccharides, were fermented in the caecum. The explanation for the increase in $\mathrm{pH}$ in distal colon and faeces induced by NSP is more problematic. The hydration and bulking properties of NSP may have diluted the acidic compounds, but our ex vivo experiments indicated that the luminal buffer capacity allows up to threefold dilutions without affecting $\mathrm{pH}$ (data not shown). The observation that $\mathrm{pH}$ was driven by SCFA in proximal regions, but not in distal colon and faeces (fig 5), strongly suggests that other metabolites, not measured in this study, are involved in distal colonic regions. A large number of compounds have been implicated as determinants of luminal $\mathrm{pH}$, including products of incomplete fermentation. ${ }^{5}$

Although NSP disappeared from digesta only proximally (fig 1), elevated concentrations of SCFA were still observed distally (fig 3). Whether proximally produced SCFA were transported to distal regions due to intestinal hurry, or whether breakdown products of the fibre were transported further distally and fermented there, cannot be concluded from this study and requires further investigation. Clearly, luminal SCFA concentrations are not simply a reflection of SCFA production, but are also affected by absorption rate and intestinal bulk. For example, the large differences in caecal amounts of SCFA (fig 3C) were masked by equally large differences in bulk, resulting in similar SCFA concentrations in all groups (fig $3 \mathrm{~A})$. We realise that the calculated flow rates of SCFA (concentration $\times$ digesta flow; fig $3 \mathrm{C}, \mathrm{D}$ ) are dependent on production and absorption rates. However, we feel that these total amounts provide an indication of the magnitude of fermentation and the absolute amounts of SCFA exposed to the mucosa. Whether the concentration of SCFA, that is, what the epithelium perceives, or the absolute amount of SCFA available, is the physiologically more relevant variable, remains to be determined. This is of particular importance to poorly fermentable substrates with strong bulking properties, such as insoluble NSP from wheat bran.

Ammonia is the major end product of bacterial fermentation of nitrogenous compounds in the large bowel. As for SCFA, the luminal ammonia concentration is the net result of production, absorption, and intestinal bulk. In addition, ammonia can be assimilated for de novo synthesis of bacterial protein. ${ }^{7}$ The effect of RS fermentation in lowering ammonia concentration (fig 4) may be mediated via several pathways. Ample carbohydrate available for 
fermentation may reduce protein fermentation, and hence ammonia production. Furthermore, increased carbohydrate fermentation may stimulate assimilation of ammonia nitrogen for bacterial growth, as was suggested by Rémésy and Demigné. ${ }^{31}$ This seems likely considering the fact that RS, in contrast to NSP, was fully fermented in the colon and therefore would support bacterial proliferation. Irrespective of the mechanism, a low ammonia concentration in the colonic lumen is desirable, as ammonia is toxic to the epithelium in high concentrations ${ }^{6}$ and has been implicated in colon carcinogenesis. $^{38}$

In research into distal luminal contents, faecal indexes have generally been assumed to reflect distal colonic conditions. This appeared to be true in rats. ${ }^{2}$ In the present study, faecal mass and excretion of starch, NSP, and SCFA, did accurately reflect those in the distal colon. For SCFA and ammonia concentrations, however, faecal results did not always accurately reflect the conditions in the distal colon. Faecal values were higher with greater variation and group means were different relative to each other, suggesting a large variation in the final dehydration of luminal contents immediately before defecation. Therefore, caution should be exercised in the interpretation of faecal results.

\section{Conclusion}

Wheat bran, a rich source of insoluble NSP, can shift the fermentation of RS further distally, resulting in favourable changes in the distal colonic luminal environment, the region where tumours most commonly occur. Therefore, the concomitant consumption of insoluble fibre and RS containing products will combine and strengthen the beneficial effects on colonic physiology of both substratesincreasing transit, increasing butyrate, and lowering ammonia - and may contribute to a dietary modulation of colon cancer risk.

This study was financially supported by Deakin University (Postdoctoral Research Fellowship to MJAPG). The author wish to thank Starch Australasia Ltd (Lane Cove, New South Wales, Australia) for kindly providing dietary ingredients. Robert Nason, Doug Kerton, Genny Power, Melissa Cameron, and Zhong Xian $\mathrm{Lu}$ are thanked for excellent assistance during slaughter, Ann McIntyre for SCFA analysis, Sing Kai Lo for advice on statistical analysis, and John Pluske for stimulating discussions. Part of these data has been published in abstract form (Altering the site of fermentation in the pig: implications ings of the Sixth Conference of the Australasian Pig Science Association, 1997;6:181).

1 Kritchevsky D. Epidemiology of fibre, resistant starch and colorectal cancer. Eur f Cancer Prev 1995;4:345-52.

2 McIntyre A, Young GP, Taranto T, et al. Different fibers have different regional effects on luminal contents of rat colon. Gastroenterology 1991;101:1274-81.

3 McIntyre A, Gibson PR, Young GP. Butyrate production from dietary fibre and protection against large bowel cancer from dietary fibre and protection agai
in a rat model. Gut 1993;34:386-91.

4 Scheppach W. Effects of short chain fatty acids on gut morpholgy and function. Gut 1994;35(suppl 1):S35-8.

5 Newmark HL, Lupton JR. Determinants and consequences of colonic luminal $\mathrm{pH}$ : implications for colon cancer. Nut Cancer 1990;14:161-73.

6 Lin HC, Visek WJ. Colon mucosal cell damage by ammonia in rats. F Nutr 1991;121:887-93.

7 Wrong OM. Bacterial metabolism of protein and endogenous nitrogen compounds. In: Rowland IR, ed. Role of the gut flora in toxicity and cancer. London: Academic Press, 1988:227-62.

8 Payler DK, Pomare EW, Heaton KW, et al. The effect of wheat bran on intestinal transit. Gut 1975;16:209-13.
9 Englyst HN, Kingman SM, Cummings JH. Classification and measurement of nutritionally important starch fracand measurement of nutritionally important starc

10 Weaver GA, Krause JA, Miller TL, et al. Cornstarch fermentation by the colonic microbial community yields more butyrate than does cabbage fiber fermentation; cornstarch fermentation rates correlate negatively with methanogenesis. Am $\mathcal{F}$ Clin Nutr 1992;55:70-7.

11 Topping DL, Illman RJ, Clarke JM, et al. Dietary fat and fiber alter large bowel and portal venous volatile fatty acids and plasma cholesterol but not biliary steroids in pigs. $\mathcal{F}$ Nutr 1993;123:133-43.

12 Marsono Y, Illman RJ, Clarke JM, et al. Plasma lipids and large bowel volatile fatty acids in pigs fed on white rice, brown rice and rice bran. Br F Nutr 1993;70:503-13.

13 Noakes M, Clifton PM, Nestel PJ, et al. Effect of high-amylose starch and oat bran on metabolic variables and bowel function in subjects with hypertriglyceridemia. Am f Clin Nutr 1996;64:944-51.

$14 \mathrm{Lu}$ ZX, Muir JG, Collier GR. Comparison of resistant tarch with soluble and insoluble non-starchpolysaccharides on faecal output and fermentation dependent events in rats. Proc Nutr Soc Australia 1995;5:41.

15 Young GP, McIntyre A, Albert V, et al. Wheat bran suppresses potato starch-potentiated colorectal tumorigenesis at the aberrant crypt stage in a rat model. Gastroenterology 1996;110:508-14.

16 Phillips J, Muir JG, Birkett AM, et al. Effect of resistant starch on faecal bulk and fermentation-dependent events in humans. Am f Clin Nutr 1995;62:121-30.

17 Birkett A, Muir JG, Phillips J, et al. Resistant starch lowers faecal concentrations of ammonia and phenols in humans. Am 7 Clin Nutr 1996;63:766-72.

18 Cummings JH, Beatty ER, Kingman SM, et al. Digestion and physiological properties of resistant starch in the and physiological properties of resistant starch

19 Shetty PS, Kurpad AV. Increasing starch intake in the human diet increases faecal bulking. Am 7 Clin Nutr 1986; 43:210-12.

20 Van Munster IP, Tangerman A, Nagengast FM. Effect of resistant starch on colonic fermentation, bile acid metabolism and mucosal proliferation. Dig Dis Sci 1994;39:83442 .

21 Key FB, Mathers JC. Gastrointestinal responses of rats fed on white and wholemeal breads: complex carbohydrate digestibility and the influence of dietary fat content. $\mathrm{Br} \mathcal{F}$ Nutr 1993;69:481-95.

22 Graham H, Aman P. The pig as a model in dietary fibre digestion studies. Scand $\mathcal{F}$ Gastroenterol 1982;22(suppl 129):55-61.

23 Miller ER, Ullrey DE. The pig as a model for human nutrition. Ann Rev Nutr 1987;7:361-82.

24 Key FB, Mathers JC. Digestive adaptations of rats given white bread and cooked haricot beans (Phaseolus vulgaris): large bowel fermentation and digestion of complex carbohydrates. Br 7 Nutr 1995;74:393-406.

25 Muir JG, O'Dea K. Validation of an in vitro assay for predicting the amount of starch escaping digestion in the small intestine of humans. Am F Clin Nutr 1993;57:540-6.

26 Englyst HN, Cummings JH. Improved method for measurement of dietary fiber as non-starch polysaccharide in plant foods. $\mathcal{F}$ Assoc Anal Chem 1988;71:808-14.

27 Lin HC, Visek WJ. Large intestinal $\mathrm{pH}$ and ammonia in rats: dietary fat and protein interactions. F Nutr 1991;121:83243.

28 Scott TA, Boldaji F. Comparison of inert markers [chromium oxide or insoluble ash (Celite)] for determining apparent metabolizable energy of wheat- or barley-based broiler diets with or without enzymes. Poult Sci 1997;76: 594-8.

29 Van Keulen J, Young BA. Evaluation of acid-insoluble ash as a natural marker in ruminant digestibility studies. 7 Anim Sci $1977 ; 44: 282-7$.

30 Goodlad JS, Mathers JC. Large bowel fermentation in rats given diets containing raw peas (Pisum sativum). Br f Nutr 1990;64:569-87.

31 Rémésy C, Demigné C. Specific effects of fermentable carbohydrates on blood urea flux and ammonia absorption in the rat caecum. F Nutr 1989;119:560-5.

32 Cassidy A, Bingham SA, Cummings JH. Starch intake and colorectal cancer risk: an international comparison. $\mathrm{Br} f$ Nutr 1994;69:937-42.

33 Topping DL, Gooden JM, Brown IL, et al. A high amylose (amylomaize) starch raises proximal large bowel starch and increases colon length in pigs. F Nutr 1997;127:615-22.

34 Heijnen ML, Beynen AC. Consumption of retrograded (RS3) but not uncooked (RS2) resistant starch shifts nitrogen excretion from urine to feces in cannulated piglets. $\mathcal{F}$ Nutr 1997;127:1828-32.

35 Goodlad JS, Mathers JC. Digestion by pigs of non-starchpolysaccharides in wheat and raw peas (Pisum sativum) fed polysaccharides in wheat and raw peas (Pis

36 Van Dokkum W, Pikaar NA, Thissen JTNM. Physiological effects of fibre-rich types of bread. 2. Dietary fibre from bread: digestibility by the intestinal microflora and water-holding capacity in the colon of human subjects. $\mathrm{Br} \mathcal{F}$ Nutr 1983;50:61-74

37 Tomlin J, Read NW. The effect of resistant starch on colon function in humans. Brf Nutr 1990;64:589-95.

38 Clinton SK, Bostwick DG, Olson LM, et al. Effects of ammonia acetate and sodium cholate on N-methyl-N'nitro-N-nitrosoguanidine-induced colon carcinogenesis of rats. Cancer Res 1988;48:3035-9. 